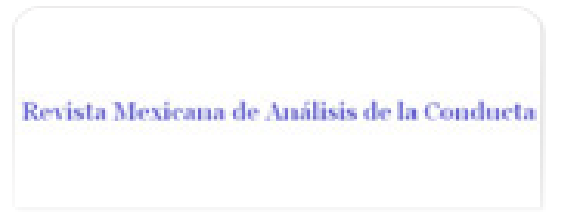

Revista Mexicana de Análisis de la Conducta ISSN: 0185-4534

editora@rmac-mx.org

Sociedad Mexicana de Análisis de la Conducta México

Valencia Ortíz, Andrómeda Ivette; Flores León, Araceli; Sánchez Sosa, Juan José Efectos de un programa conductual para el cuidado de pacientes oncológicos pediátricos Revista Mexicana de Análisis de la Conducta, vol. 32, núm. 2, diciembre, 2006, pp. 179-198 Sociedad Mexicana de Análisis de la Conducta Guadalajara, México

Disponible en: http://www.redalyc.org/articulo.oa?id=59332206

Cómo citar el artículo

- Número completo

- Más información del artículo

Página de la revista en redalyc.org

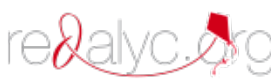

Sistema de Información Científica Red de Revistas Científicas de América Latina, el Caribe, España y Portugal Proyecto académico sin fines de lucro, desarrollado bajo la iniciativa de acceso abierto 


\title{
EFECTOS DE UN PROGRAMA CONDUCTUAL PARA EL CUIDADO DE PACIENTES ONCOLÓGICOS PEDIÁTRICOS*
}

\author{
EFFECTS OF A BEHAVIORAL INTERVENTION TO IMPROVE THE CARE \\ OF CHILDREN WITH CANCER
ANDRÓMEDA IVETTE VALENCIA ORTÍZ, ARACELI FLORES LEÓN Y JUAN JOSÉ SÁNCHEZ SOSA. \\ UNIVERSIDAD NACIONAL AUTÓNOMA DE MÉXICO
}

\begin{abstract}
Recent developments in medical treatment allow for approximately $70 \%$ of children with cancer to survive. In developing countries such as Mexico, however, the oncological disease still represents the two main causes of death in children 6 to 14 years old. Due to medication side-effects and the invasive nature of some medical procedures, cancer's development and treatment sharply decrease the quality of life of patients and their immediate relatives and may decrease the effectiveness of health care. The purpose of the present study was to examine the effects of a behavioral intervention aimed at establishing self-care behaviors, social skills to facilitate treatment and general improvement of quality of life. The intervention involved such procedures as modeling, shaping, positive reinforcement and behavioral essays. The experimental comparisons involved individual replications with six boys 5 to 15 years old and their immediate relatives. Measurement included a behavioral interview, medical treatment adherence, behavioral recording systems, and verbal measures such as a social satisfaction questionaire, evaluation of the treatment components, the Pediatric Quality of Life Inventory, and the Content Analysis of Verbatim Explanations. The intervention focused on the modifica-

* $\quad$ El presente estudio fue posible, en parte, gracias al financiamiento IN-309705 de la Universidad Nacional Autónoma de México y a otros apoyos de la Unión Internacional de Psicología Científica y de la División de Psicología Clínica y Comunitaria de la International Association of Applied Psychology. Una versión previa constituyó la tesis doctoral del primer autor bajo la dirección del último. Los autores agradecen de manera especial la contribución del Dr. Héctor Ayala a una versión preliminar del programa de intervención.
\end{abstract}


tion or establishment of hygiene, nutrition and treatment-related behaviors. Procedures also involved modifying intra-verbal behaviors such as pessimistic thoughts into positive ones, as well as social skills training (assertive comunication and negotiation) and problem-solving skills. The intervention consisted of seven training units for the parents and six units for the children and their siblings. Results showed clinical and statistically significant changes in all target behaviors, and increased quality of life perception. Results are discussed in the context of scarcity of resources conditions usually prevalent in developing countries.

Key Words: cancer, terapeutic adherence, quality of live, social skills, behavior modification.

\section{RESUMEN}

Los avances en el tratamiento médico permiten que el $70 \%$ de niños con cáncer sobrevivan, sin embargo en México los padecimientos oncológicos aún se encuentran entre las primeras causas de muerte en niños entre los 6 y 14 años de edad. El desarrollo del cáncer y su tratamiento disminuyen la percepción de la calidad de vida y generan en los niños alteraciones en las conductas de autocuidado. El propósito del presente estudio fue evaluar los efectos de un programa de modificación cognitivo-conductual para el establecimiento de destrezas de autocuidado, habilidades sociales y mejora de la calidad de vida, mediante procedimientos tales como modelamiento, moldeamiento, reforzamiento positivo y ensayo conductual en niños con cáncer. Las comparaciones experimentales incluyeron seis réplicas individuales de los efectos en niños entre 5 y 15 años y sus parientes inmediatos. El estudio utilizó una entrevista conductual, sistemas de autoregistro de adherencia al tratamiento médico y medidas verbales tales como un cuestionario de satisfacción social, una evaluación general sobre los componentes del tratamiento un cuestionario de calidad de vida pediátrica y la técnica de análisis del contenido verbal de creencias y explicaciones. El programa se enfoca en la modificación de conductas de higiene, alimentación, y otras relacionadas con el tratamiento médico, modificación de conductas intra-verbales pesimistas en optimistas, entrenamiento en comunicación asertiva y negociación y solución de problemas. El programa consiste en siete módulos de entrenamiento para los padres y seis para los niños y sus hermanos. Los resultados mostraron cambios clínicos y estadísticamente significativos para las conductas blanco, así como una mejora en la percepción de la calidad de vida. Los resultados se discuten en el contexto de condiciones de escasez de recursos usualmente prevalentes en países en desarrollo.

Palabras clave: cáncer, modificación conductual, calidad de vida, adherencia terapéutica, habilidades sociales. 
En México los padecimientos oncológicos en niños se encuentran entre las sus primeras causas de defunción. Actualmente, los avances médicos permiten que alrededor del $70 \%$ de los niños que padecen cáncer sobrevivan si reciben tratamiento oportuno y adecuado. El cáncer es una enfermedad que se caracteriza por la incapacidad celular de detener su proliferación debido a la inactividad del mecanismo apoptótico (muerte celular programada) y la capacidad de convertirse en células malignas dispersándose a otros órganos o tejidos en el proceso conocido como metástasis (Baum \& Andersen, 2001).

El diagnóstico de cáncer y el desarrollo de la enfermedad son generalmente inesperados y funcionan como fuente de estrés que provoca alteraciones biológicas y cambios en las conductas relacionadas con la salud. Se disminuye la percepción de la calidad de vida, lo que puede predisponer a la enfermedad o a exacerbar el carácter de su diagnóstico (Baum \& Andersen, 2001). Esta situación genera en los niños y en sus familiares cambios emocionales y de comportamiento, como la desesperanza, depresión y soledad (Barón \& Ordoñez, 1998; Friedman \& Mulhem, 1992).

El afrontamiento a la enfermedad por los niños y sus padres se basa en las habilidades que tengan para resistir eficazmente al estresor lo que se ha denominado "fortaleza psicológica" (Kobasa, 1979). Se han documentado algunos factores psicológicos relacionados con las respuestas ante padecimientos oncológicos (Green \& Shellenberger, 1991) entre los que se incluyen: 1) factores conductuales (seguimiento de instrucciones, "indefensión" o depresión la fatiga y el nivel de actividad física; 2) factores emocionales (supresión de emociones negativas); 3 ) factores intraverbales o cognitivos (pensamientos pesimistas) y 4) factores sociales (aislamiento, carencia de apoyo social y déficit en habilidades sociales), que conforman lo que otros autores han denominado personalidad "Tipo C" (Anarte, López, Ramírez \& Esteve, 2000; Morris \& Creer, 1980; Temoshok, 1987).

Las conductas que se observan en las personas que muestran indefensión se han asociado reiteradamente con la disminución de la adherencia terapéutica y con la presencia de alteraciones en el funcionamiento del sistema inmune tales como la disminución de las células $T$ y alteraciones en el funcionamiento de las células NK (natural killer) (Bartrop, Lockhurts, Lazarus, Kiloh \& Penny, 1977; Burns \& Seligman, 1989; Irwin, Daniels, Bloom, Smith \& Weiner, 1987; Kiecolt-Glaser, 1991; Peterson, Seligman \& Vaillant, 1988; Wayner, Cox, \& Mackay, 1979).

La importancia de estos hallazgos radica en que las personas "pesimistas" se encuentran en riesgo de desarrollar o exacerbar alteraciones en su sistema inmune, presentar enfermedades o complicar su estado de salud al mostrar baja adherencia trerapéutica. Es posible que esta serie de eventos se asocien con que los pacientes no perciben que su comportamiento cambia su 
salud. Un ejemplo de lo anterior se ilustra con hallazgos que muestran que las personas con pensamientos pesimistas se enferman dos veces más en comparación con las personas optimistas (Peterson, Seligman \& Vaillant, 1988).

De igual forma, la indefensión o sensación de impotencia suele relacionarse con la forma en que las personas explican el origen de las cosas que les suceden. Seligman denominó a esto "estilo explicativo" y propone tres dimensiones que suelen utilizarse para explicar la ocurrencia de cualquier suceso positivo o negativo: 1) dimensión de permanencia, se refiere al contraste entre una creencia temporal vs. una convicción relativamente permanente. Los niños que están en riesgo de una depresión caracterizan las causas de los sucesos negativos que les ocurren como permanentes. 2) dimensión de dominio, se refiere a convicciones específicas vs. globales. Los niños que informan que una causa es permanente y global identificarán sus efectos a lo largo del tiempo, mostrando conductas como darse por vencidos en múltiples áreas y no sólo en la relativa al problema y 3 ) dimensión de dominio personal, el cual alude a pensamientos referentes a quién es responsable de un error o problema, en este caso los niños pueden verbalizar que se culpan a sí mismos y mostrando índices bajos en escalas verbales de autoestima (Abramson, Seligman \& Teasdale, 1978; Sweeney, Anderson \& Bailey, 1986; Weiner, Frieze, Kukla, Reed, Rest \& Rosenbaum, 1971).

La combinación de verbalizaciones o pensamientos globales, permanentes e internos suelen llevar a los individuos a tener pensamientos pesimistas, mientras que la combinación de verbalizaciones temporales, específicas y externas son característicos de las personas "optimistas". Investigaciones en niños con altos niveles de síntomas depresivos coinciden en señalar que éstos tienen un estilo pesimista para explicar los eventos (Altman \& Gotlib, 1988 , Hops, 1992, 1995) y por lo tanto muestran déficits en habilidades sociales y bajo dominio para resolver problemas, incluyendo los de salud.

Seligman, Reivich, Jaycox y Gillham (1996) han propuesto que los estilos explicativos surgen desde la infancia moldeados por el estilo explicativo de la madre, por las críticas de los adultos significativos (padres y maestros), y por la forma en la que se manejan las situaciones de crisis (por ejemplo, una enfermedad crónica) es decir, si ante la crisis presentan estilos optimistas o pesimistas (Dweck \& Licht, 1980; Seligman \& Elder, 1985).

La importancia de estos hallazgos radica en que las personas pesimistas se encuentran en mayor riesgo de padecer alteraciones en su sistema inmune, presentar enfermedades y complicar su estado de salud al tener baja adherencia médica al no percibir que su comportamiento puede afectar su salud (Sánchez-Sosa, 2002a). Estudios sobre morbilidad y mortalidad a corto, mediano y largo plazo señalan que la falta de adherencia terapéutica del paciente con cáncer interfiere y genera estadísticas falsas y poco confiables (Aaronson, 1990; Donovan, Sanson-Fisher \& Redman, 1989). 
Por otra parte, las personas optimistas resisten la indefensión, no se deprimen tan fácilmente y por lo tanto reducen la probabilidad de que su sistema inmune altere su funcionamiento. Estos individuos se enferman menos y buscan ayuda profesional cuando se presenta alguna alteración en su salud, muestran mayor adherencia terapéutica y despliegan habilidades sociales que les permiten conseguir apoyo social en momentos difíciles; lo cual contribuye a amortiguar el impacto del estrés. Finalmente, suelen ser personas que saben resolver problemas por lo que se perciben competentes y exitosos en diversos escenarios como los familiares, académicos y laborales (Aspinwall \& Brunhart, 2000; Aspinwall, Richer \& Hoffman, 2001; Danner, Snowdon, Friesen, 2001; Fredrickson, 1998, 2001; Headey \& Wearing, 1989; Isen, Rosenzweig \& Young, 1991; Kamen-Siegel, Rodin, Seligman \& Dwyer, 1991; Maruta, Colligan, Malinchoc \& Offord, 2000; Ostir, Markides, Black \& Goodwin, 2000; Staw, Sutton, Pelled, 1994; Seligman, 1998, 2002; Segerstrom, Taylor, Kemeny \& Fahey, 1998; Valliant, 2000, 2002).

Otro factor relacionado con la adaptación de los niños al cáncer es la autoeficacia percibida, es decir, el grado de convicción de un paciente de ser capaz de exhibir las conductas requeridas para producir resultados que se reflejen en la adherencia terapéutica. Cada conducta a realizar implica la elección de cuánto esfuerzo debe emplear el paciente y durante cuánto tiempo deberá afrontar situaciones que le produzcan tensión (Bandura, 1977), si se interesa en el rol de los factores intraverbales o cognitivos (percepción y juicio) y cómo éstos afectan a las emociones y la conducta (Sánchez-Sosa, 2002b).

Al igual que la autoeficacia, el apoyo social es un predictor del estado de salud, de la mortalidad y de la adaptación ante acontecimientos estresantes. Los individuos con escasos lazos sociales presentan menos adherencia al tratamiento y el impacto de la enfermedad es mucho más estresante (House, Landis, Umberson, 1998). Otros estudios han sugerido que es la calidad del apoyo social, más que su cantidad la que predice la adherencia en pacientes crónicos (Sheridan \& Ratmaches, 1992).

En el caso de los niños, las diferencias individuales en el desarrollo, el tipo de conductas, la responsividad al tratamiento y la calidad de la relación entre el niño y al menos uno de sus padres son variables que afectan la adherencia y modulan el riesgo en contraste con una familia con interacción inadecuada (Repetti, Taylor \& Seeman, 2002; Rutter, 1978).

Así, debido a los múltiples efectos que el comportamiento muestra sobre enfermedades como el cáncer y el malestar que éstas originan en los niños y sus familias es importante contar con estrategias de intervención psicológica que permitan desarrollar conductas para adaptarse al proceso y mejorar la calidad de vida del paciente y su familia (Bayés, 1990 a,b).

Las intervenciones cognitivo-conductuales han mostrado efectos en la mejoría del repertorio de autocuidado y el incremento de habilidades socia- 
les. Estas estrategias también cambian los estilos explicativos y afectan el funcionamiento inmune con el consecuente aumento de la probabilidad de mejorar el estado de salud de los pacientes o incluso quizá la prevención de futuras complicaciones asociadas con el desajuste emocional (Antoni, Cruess, Cruess, Lutgendorf, Kumar, Ironson, Klimas, Fletcher, \& Schneiderman, 2000; Antoni, Cruess, Cruess, Kumar, Lutgendorf, Ironson, Dettmer, Williams, Klimas, Fletcher, \& Schneiderman, 2000; Bartrop, Lockhurts, Lazarus, Kiloh, \& Penny, 1977; Beck, 1976; Beck, Rush, Shaw \& Emery, 1979; Cruess, Antoni, Cruess, Fletcher, Ironson, Kumar, Lutgendorf, Hayes, Klimas \& Schneiderman, 2000; DeRubeis, Evans, Hollon, Garvey, Grove \& Tuason, 1990; Elkin, Pilkonis, Docherty \& Sotsky, 1988; Ellis, 1962; Galán, 1995; Green, Green \& Santoro, 1988; Glaser \& Kielcolt-Glaser, 1985; Kazdin \& Wilcoxon, 1976; Kuhl, 1981; Nolen-Hoeksema, 1990; Vecina, 2006). Así, al instrumentar un programa de modificación cognitivo-conductual en niños con cáncer y sus familias se espera que los participantes incrementen conductas de adherencia terapéutica, mejoren su percepción sobre su calidad de vida e incrementen sus habilidades sociales y cognitivas.

\section{MÉTODO}

\section{Participantes}

Participaron seis varones con cáncer con edades entre los 5 y 15 años y sus familias ( $N=19$ participantes). El diagnóstico de cuatro de los niños fue de leucemia aguda linfoblástica (LAL), uno de Castelman multicéntrico y otro de germinoma comórbido con diabetes. Uno de los niños no asistía a la escuela al momento de iniciar el estudio, dos estaban en primaria y tres en secundaria. Participaron cuatro padres (30-50 años de edad), cinco madres (29-39 años de edad), y cuatro hermanos (7-25 años de edad). El nivel socioeconómico de las familias se clasificó como bajo en cinco de los casos y medio en uno. Los niños se seleccionaron de una lista de pacientes proporcionada por una institución de asistencia privada para niños con cáncer. A fin de fomentar la viabilidad del estudio se eligieron niños con cáncer que tuvieran de 5 a 15 años de edad y radicaran en el D. F. De esta lista aceptaron participar en el estudio seis niños. Para iniciar su participación niños y padres firmaron un consentimiento informado.

\section{Escenario}

Centro de Servicios Psicológicos en la Facultad de Psicología, UNAM. Los cubículos en los que se trabajó tenían una dimensión de $3 \times 3 \mathrm{~m}$. y contaban 
con tres sillones o sillas y una mesa de trabajo. Todos los cubículos tenían iluminación y ventilación adecuada.

\section{Medición}

Carnets de adherencia al tratamiento médico (Flores \& Bazán, 2004). Es un Instrumento de autorregistro en forma de cuadernillo en que se asientan las conductas de adherencia del niño, tales como: la asistencia al hospital para tratamiento, consultas adicionales, molestias y recomendaciones de dieta e higiene (lavarse los dientes y manos, bañarse, etc.). Las conductas blanco de adherencia se registraron en términos de porcentaje dividiendo el número de conductas de adherencia que realizan los niños cada semana entre el número de conductas de adherencia que debe realizar cada niño de acuerdo con las indicaciones dadas por el médico para dicho período y multiplicadas por 100. Las conductas de adherencia se tomaron de la lista de recomendaciones dadas por el médico al inicio del tratamiento médico.

Cuestionario de Satisfacción Social con los Resultados del Tratamiento (Valencia, 2006). El cuestionario evalúa la satisfacción de los usuarios con respecto a las habilidades que adquirieron al terminar el tratamiento. El cuestionario de los niños contuvo 13 preguntas y tres opciones de respuesta (no, no lo sé y sí). El cuestionario de los padres contuvo 28 reactivos con cinco opciones de respuesta que van desde "completamente de acuerdo" hasta "completamente en desacuerdo". El instrumento fue validado por jueces expertos en el área de salud. Si un reactivo del instrumento no recibía un mínimo de $80 \%$ de acuerdo independiente respecto a su pertinencia a la conducta en cuestión, su contenido se revisaba hasta lograrlo con nuevos jueces, también independientes o el reactivo se descartaba.

Entrevista Conductual (Valencia, 2006). Consta de una ficha de identificación y 54 preguntas sobre características sociodemográficas de la familia, el área de la salud del niño, el área de crianza, actividades en el hogar y actividades en la escuela. Las preguntas están enfocadas al análisis funcional e identificación de parámetros conductuales (frecuencia, y duración de la conducta).

Evaluación general sobre los componentes del tratamiento (eg- valencia, 2006). Consta de 19 afirmaciones que describen el comportamiento que se espera del paciente de acuerdo con los objetivos de la intervención denominada "Juego del Optimismo". Se incluyen aspectos sobre adherencia al tratamiento, reestructuración intraverbal o cognitiva, solución de problemas, habilidades sociales y seguimiento de instrucciones. Se identificaron estos comportamientos antes y después del tratamiento, analizando cada respuesta en una escala tipo Likert con cinco opciones de respuesta que van desde completamente de acuerdo, hasta completamente en desacuerdo. 
Cuestionario de Calidad de Vida Pediátrica (Pediatric Quality if Life Inventory -PedsQL- Varni, y cols, 1998, revisado y adaptado por Cantú, Cordero \& Chávez, 2003). Se utilizaron las versiones 3.0 y la 4.0 del PedsQL, ambas incluyen un informe para niños y uno para sus padres, los mismos ítems son evaluados por ambos. Las dos versiones tienen una división según la edad: un registro para 5 a 7 años de edad y otro para 8 a 12 . El PedsQL es un cuestionario de preguntas cerradas con opciones de respuesta en escala Likert de cinco intervalos para los padres y los niños de 8 a 12 años de edad y tres para los niños de 5 a 7 años. La versión 4.0 valora la calidad de vida en general y puede aplicarse a cualquier niño independientemente de su condición de salud. Consta de las siguientes cuatro dimensiones sobre diferentes áreas de la vida del niño: a) funcionamiento físico (8 ítems), b) funcionamiento emocional (5 ítems), c) funcionamiento social (5 ítems), y d) funcionamiento escolar (5 ítems). La versión 3.0 versa sobre la calidad de vida relacionada con la salud, específicamente a los problemas relativos al cáncer y consta de las siguientes ocho dimensiones: a) dolores y molestias (2 ítems), b) náusea (5 ítems), c) procedimientos médicos (3 ítems), d) tratamientos (3 ítems), e) preocupaciones (3 ítems), f) problemas cognitivos (5 ítems), g) apariencia física ( 3 ítems) y h) comunicación (3 ítems).

Técnica de análisis del contenido verbal de las explicaciones (Content Analysis of Verbatim Explanations Technique- CAVE- Schulman, Castellon \& Seligman, 1989). Se utilizó la técnica de análisis del contenido verbal de las explicaciones "CAVE" propuesto por Schulman, Castellon y Seligman (1989), como una estrategia pertinente a la exploración del "estilo atribucional" de los niños y sus padres. Para el uso del CAVE se requiere que la persona evaluada mencione a qué atribuye las situaciones que se le presentan (por qué cree que ocurre eso), se registran sus respuestas y posteriormente jueces expertos e independientes evalúan por separado los contenidos explicativos de cada verbalización, identificando la respuesta dentro de cada una de tres dimensiones: a) personalización (interna vs externa), b) permanencia (temporal vs. permanente) y c) de dominio (específico vs. global). Para verificar el estilo explicativo de los niños y sus padres se diseñaron tarjetas con 10 viñetas con situaciones para los niños y 10 situaciones similares para los padres. La aplicación es individual presentando cada tarjeta y preguntando ¿Por qué cree que le ocurre eso (cada situación)?, registrando todas las verbalizaciones. Posteriormente, se les vuelve a presentar cada situación pero ahora se les muestran tres explicaciones (de acuerdo a las tres dimensiones de personalización, permanencia y dominio) y se les pide que mencionen cómo se sentirían con cada una de esas explicaciones (consecuencia emocional) y se registran sus respuestas. 


\section{Procedimiento}

Una vez firmado el Consentimiento informado, se les explicaba a niños y padres por separado la manera de llenar los carnets de adherencia. Los niños recibían instrucciones para marcar en su carnet las actividades realizadas diariamente, de acuerdo con las indicaciones del médico, tales como la dieta, acudir al hospital, conductas de higiene etc. Los padres recibían indicaciones para marcar en el carnet si sus hijos habían realizado las actividades indicadas por el médico. Estos carnets se entregaban y recolectaban semanalmente hasta concluir el tratamiento. Las primeras tres semanas de registro correspondían al periodo de línea base.

Durante la fase de evaluación se aplicó la entrevista conductual, el PedsQL, la EG y se instrumentó la técnica CAVE.

Al término de la fase de evaluación se dio inicio con el tratamiento "El Juego del Optimismo" conformado por cinco módulos para los niños, seis para los padres y uno introductorio para ambos. Los participantes podían avanzar a un nuevo módulo sólo cuando cumplían con el criterio de adquisición de habilidades.

Conductas blanco y criterios de adquisición para cada modulo:

1. Módulo de Introducción: Informar al niño y al padre sobre la enfermedad, los tratamientos médicos y la importancia de seguir las recomendaciones médicas, instruir en el uso adecuado de los carnets de auto-registro de las conductas de adherencia terapéutica. El criterio fue que el niño utilizara adecuadamente el auto-registro, describiera las características de su enfermedad y realizara las siguientes conductas de adherencia:

a) Conductas de higiene: Lavarse las manos por lo menos tres veces al día (antes de cada comida), bañarse todos los días, lavarse los dientes tres veces al día (después de cada comida).

b) Conductas de Alimentación: Realizar tres comidas al día, ingiriendo aquellos alimentos que beneficien a su salud, recomendados por el médico. El criterio aceptable es que el niño realice tres comidas al día.

c) Conductas dirigidas al tratamiento médico: Asistir a todas sus consultas médicas los días indicados por el médico tratante y a todos los estudios médicos que se requieran, así como exponerse al total de sus ciclos de tratamiento. Tomar sus medicamentos en las dosis y horas indicadas por el médico y asistir a consultas médicas cuando tenga molestias.

2. Identificar el pensamiento automático (Módulo 1): Los participantes deben verbalizar la creencia inicial ante una situación y su consecuencia emocional. El criterio de adquisición es que el participante verbalice o 
escriba los componentes del modelo $A B C$ (adversidad, creencia y consecuencia) en el $100 \%$ de los ejercicios de práctica de este módulo.

3. Cambiar el estilo de pensamiento (Módulo 2): Los participantes deben, después de identificar su estilo explicativo cambiarlo, en su caso, de pensamiento pesimista a optimista. El criterio de adquisición es que el participante verbalice o escriba el cambio de su estilo de pensamiento pesimista a optimista en el $100 \%$ de los ejercicios de práctica de este módulo.

4. Quitar el desastre (Módulo 3): Los participantes deben adquirir habilidades para descatastrofizar (no interpretar eventos o molestias como catastróficas). El criterio de adquisición es que el participante verbalice o escriba alternativas de pensamiento que cambien consecuencias emocionales y de comportamiento negativas en el $100 \%$ de los ejercicios de práctica de este módulo.

5. Solucionar problemas (Módulo 4): Los participantes deben aprender y poner en práctica habilidades de solución de problemas. El criterio de adquisición es que el participante verbalice o escriba la solución a diferentes problemas en el $100 \%$ de los ejercicios de práctica de este módulo.

6. Habilidades para relacionarnos mejor (Módulo 5): Los participantes deben aprender y poner en práctica habilidades de comportamiento pro-social, es decir, conducta de comunicación asertiva y de negociación. El criterio de adquisiciones que el participante verbalice o escriba conductas de comunicación asertiva y de negociación en el 100\% de los ejercicios de práctica de este módulo.

7. Taller a padres (Módulo 6, exclusivo para los padres): Los padres deben aprender: a) el análisis funcional de la conducta a través del uso de registros ACC (antecedente, conducta y consecuencia), b) establecer habilidades de seguimiento instruccional y c) los principios para la modificación de conductas inadecuadas en sus hijos (establecimiento de reglas, uso y pérdida de privilegios, etc.). El criterio de adquisición es que el participante escriba o verbalice la conducta meta de modificación, y establezca reglas para el uso y pérdida de privilegios que modifiquen conductas inadecuadas en sus hijos, en el $100 \%$ de los ejercicios de práctica de este módulo.

8. Cierre del Tratamiento: al terminar el tratamiento se aplican nuevamente los instrumentos (a excepción de la entrevista) y el cuestionario de satisfacción con los resultados de tratamiento. El niño y sus padres reciben una constancia por haber concluido el Juego del Optimismo en una pequeña ceremonia al final de la sesión.

Las estrategias conductuales para reforzar el incremento de las conductas blanco incluyeron: a) la construcción de una lista de reforzadores (para cada niño), b) reforzamiento positivo (calcomanías que se entregan al final de 
la sesión, el criterio para la entrega es que el niño haya realizado las tareas y actividades de la sesión), c) modelamiento, mediante historietas incluidas en los manuales, se buscó que el niño imitara las conductas de los personajes de cada historieta y d) ensayo conductual (se practicaron habilidades de comunicación asertiva y negociación). Cada sesión fue de 60 min. y el terapeuta en todas las ocasiones inició con la consigna de establecer rapport.

\section{RESULTADOS}

Para realizar el análisis estadístico de los datos de las conductas de adherencia terapéutica, se realizó una autocorrelación y se encontró que los datos presentaban dependencia serial. Por lo tanto, se seleccionó la prueba "técnica de división en mitades" (Split-Middle-Technique, Kazdin, 1982). Esta técnica permite examinar la tendencia o pendiente dentro de las fases de línea base e intervención y comparar las pendientes a lo largo de las fases, para determinar si hay cambios significativos entre los datos de la línea base y la intervención. La tabla 1 muestra los datos de adherencia terapéutica para los participantes.

En el caso 1 el promedio de conductas de adherencia en la LB fue de $43.96 \%$, mientras que el promedio de conductas durante el tratamiento fue de $74.17 \%$, obteniendo una ganancia de $30.21 \%$. Así, no hay cambio en el nivel, pero sí en la pendiente de la fase de LB a la fase de intervención, con una $p$ asociada de 0.0001 , lo que indica un aumento significativo en las conductas de adherencia al tratamiento médico.

En el Caso 2 el promedio de conductas, en la línea base fue de $53.05 \%$ y el promedio obtenido en la intervención fue de $71.5 \%$ con una ganancia de $18.45 \%$. Hubo un cambio significativo de nivel y pendiente de la fase de línea base a la fase de intervención con probabilidad asociada de 0.0001 .

Para el Caso 3 el promedio de conductas de adherencia en la línea base fue de $58.93 \%$ y en la intervención de $66.50 \%$. con ganancia de $7.57 \%$. Se observa un cambio de nivel y pendiente de la fase de línea base a la fase de intervención con $\mathrm{p}$ asociada de 0.0001 , al incremento de conductas de adherencia al tratamiento médico.

En el Caso 4 la adherencia durante la línea base fue de $60.46 \%$ y durante la intervención fue de $93.33 \%$. La ganancia obtenida fue de $32.87 \%$ reflejando un cambio significativo del nivel y la pendiente de la fase de línea base a la fase de intervención, también con una $p$ asociada de 0.0001 .

En el caso 5 se observa que el promedio de conductas de adherencia en la LB fue de $88.3 \%$ y durante el tratamiento fue de $92.45 \%$, con una ganancia del $4.15 \%$. Se observa cambio en el nivel y en la pendiente de LB a la fase de intervención, con nivel de significancia $p=0.0002$.

Finalmente, en el caso 6 se observa que el promedio de conductas de ad- 
herencia en la LB fue de $95.3 \%$, mientras que el promedio de conductas durante el tratamiento fue de $90.1 \%$. Se observa cambio en el nivel y en la pendiente de la LB a la fase de intervención con nivel de significancia de $p=0.0004$.

Tabla 1. Porcentaje de conductas de adherencia terapéutica antes y después del tratamiento.

\begin{tabular}{ccccc}
\hline Caso & L.B. & Tx & Ganancia & p. \\
\hline 1 & 43.96 & 74.17 & 30.21 & 0.0001 \\
\hline 2 & 53.05 & 71.5 & 18.45 & 0.0001 \\
\hline 3 & 58.93 & 66.50 & 7.57 & 0.0001 \\
\hline 4 & 60.46 & 93.33 & 32.87 & 0.0001 \\
\hline 5 & 88.3 & 92.45 & 4.15 & 0.0002 \\
\hline 6 & 95.3 & 90.1 & -5.2 & 0.0004 \\
\hline
\end{tabular}

Para evaluar la percepción de la calidad de vida antes y después del tratamiento en los seis niños y sus padres, dado el nivel de medida de las escalss, se utilizó la prueba de Wilcoxon y se encontraron diferencias estadísticamente significativas $(z=-2.207, p>0.027)$. Con un aumento de la calidad de vida percibida después del tratamiento. En Caso 1 mostró un porcentaje de ganancia de 4.4 para el niño y 7.6 para el padre. Asimismo hubo una ganancia de 11.8 para el niño y de $7.6 \%$ para el padre, en el Caso 2 . El porcentaje de ganancia del niño del Caso 3 es de $5.6 \%$, y el padre obtiene una ganancia del $16 \%$. En el Caso 4 la ganancia del niño es de $8 \%$ y para el padre es de $11.2 \%$. En el Caso 5 la ganancia en la percepción de calidad de vida del niño fue de $6.4 \%$ y la del padre de $10.8 \%$; como se observa en la Tabla 2 . Finalmente, en el Caso 6 la ganancia que se obtiene en la variable de calidad de vida es de $5.6 \%$ para el niño y $5.2 \%$ para el padre.

Las habilidades y conductas que favorecen los componentes del tratamiento se evaluaron por el terapeuta, identificando las conductas y habilidades con las que inició y terminó el Juego del Optimismo para cada niño. Se utilizó la prueba de Wilcoxon y se encontraron diferencias estadísticamente significativas en la evaluación de los componentes generales antes y después del tratamiento, $z=-2.201, p>0.028$. En todos los casos se observa un incremento de las conductas y habilidades después del tratamiento. Para el caso 1 porcentajes de ganancia fueron 56.8\%; para el Caso 2 de $65.2 \%$, para el Caso 3 fue de $73.7 \%$; en el Caso 4 de 61.1\%; en el Caso 5 de $29.5 \%$ y finalmente, el Caso 6 mostró un porcentaje de ganancia de $69.5 \%$ como muestra la Tabla 3. 
Tabla 2. Percepción de la calidad de vida antes y después del tratamiento.

\begin{tabular}{ccccccc}
\hline Caso & Pre & Post & $\begin{array}{c}\text { Ganancia } \\
\text { Niños }\end{array}$ & Pre & Post & $\begin{array}{c}\text { Ganancia } \\
\text { Padres }\end{array}$ \\
\hline 1 & 58 & 62.4 & 4.4 & 64.8 & 72.4 & 7.6 \\
\hline 2 & 63.6 & 75.4 & 11.8 & 65.2 & 72.8 & 7.6 \\
\hline 3 & 64.8 & 70.4 & 5.6 & 52 & 38 & 16 \\
\hline 4 & 52 & 60 & 8 & 56.4 & 67.6 & 11.2 \\
\hline 5 & 61.6 & 68 & 6.4 & 51.2 & 62 & 10.8 \\
\hline 6 & 60.8 & 66.4 & 5.6 & 62.8 & 68 & 5.2 \\
\hline
\end{tabular}

Tabla 3. Evaluación de los componentes del tratamiento.

\begin{tabular}{cccc}
\hline Caso & Pre & Post & Ganancia \\
\hline 1 & 34.7 & 91.5 & 56.8 \\
\hline 2 & 24.2 & 89.4 & 65.2 \\
\hline 3 & 26.3 & 100 & 73.7 \\
\hline 4 & 38.9 & 100 & 61.1 \\
\hline 5 & 67.3 & 96.8 & 29.5 \\
\hline 6 & 21 & 90.5 & 69.5
\end{tabular}

Todos los participantes, niños y padres, cambiaron sus conductas intraverbales "pensamientos pesimistas en optimistas" en el 100\% de los ejercicios de práctica. Además, los niños mostraron mejorías después del tratamiento en el número de aciertos al identificar respuestas emocionales ante dilemas de lo que podían realmente hacer para resolver un problema. Las diferencias fueron estadísticamente significativas con la prueba de Wilcoxon, $z=-2.214, p>0.027)$. De igual forma, se dieron cambios significativos en el número de aciertos de los padres $(z=-2.201, p>0.028)$ antes y después del tratamiento. En todos los casos se incrementó el número de aciertos después del tratamiento. El porcentaje de ganancia para el Caso 1 fue de $20 \%$ en el niño y del $40 \%$ para el padre; para el niño del Caso 2 la ganancia fue de $30 \%$ y para el padre de $43 \%$; en el Caso 3 fue de $13.3 \%$ para el niño y $21.7 \%$ para su padre; en el Caso 4 el porcentaje de ganancia fue del $30 \%$ tanto para el niño como el padre. En el Caso 5 la ganancia para el niño fue del $40 \%$ y para el padre del $31.6 \%$. En el Caso 6 la ganancia fue de $40 \%$ para el niño y de $37 \%$ para el padre, como se puede observar en la Tabla 4.

La validación social sobre el acuerdo con las metas del tratamiento, reveló que el caso 6 presentó un porcentaje de acuerdo del $87 \%$, seguido del caso 5 donde el porcentaje fue de $90 \%$, para el caso 1 y 3 el porcentaje fue del $96 \%$, para el caso 4 el acuerdo fue del $97 \%$ y finalmente, en el caso 2 el acuerdo 
fue del $100 \%$. En cuanto al porcentaje de satisfacción con los resultados se encontró que tres de los participantes tienen más del $90 \%$ de satisfacción y los otros tres presentaron el $100 \%$ de satisfacción con los resultados.

Tabla 4. Porcentaje de mejoría en las conductas intraverbales antes y después del tratamiento.

\begin{tabular}{ccccccc}
\hline Caso & Pre & Post & $\begin{array}{c}\text { Ganancia } \\
\text { Niños }\end{array}$ & Pre & Post & $\begin{array}{c}\text { Ganancia } \\
\text { Padres }\end{array}$ \\
\hline 1 & 70 & 90 & 20 & 40 & 80 & 40 \\
\hline 2 & 50 & 80 & 30 & 30 & 73 & 43 \\
\hline 3 & 60 & 73.3 & 13.3 & 38.3 & 60 & 21.7 \\
\hline 4 & 50 & 80 & 30 & 40 & 70 & 30 \\
\hline 5 & 50 & 90 & 40 & 31.6 & 66.6 & 31.6 \\
\hline 6 & 30 & 70 & 40 & 38 & 75 & 37 \\
\hline
\end{tabular}

\section{Discusión}

Dado que el cáncer es una fuente de estrés que impacta de manera negativa al niño que lo padece y a su ambiente familiar, y que este impacto se refleja en autocuidado deficiente y menor calidad de vida (Baum \& Andersen, 2001) el propósito del presente estudio fue diseñar y evaluar una intervención sistemática orientada a mejorar las conductas de autocuidado en el niño canceroso y su familia. Un segundo propósito consistió en mejorar su calidad de vida, tal y como la perciben los participantes.

Los participantes del presente estudio redujeron alteraciones en su vida cotidiana relacionadas con el cáncer después de la intervención. Las medidas de autoinforme, diseñadas para minimizar sesgos o la inducción artificial de respuestas, mostraron cambios clínicos y estadísticamente significativos en la calidad de vida de los niños, principalmente en la capacidad para realizar actividades sociales, físicas y escolares.

En lo relativo a las conductas de autocuidado y adherencia terapéutica, se dieron diferencias significativas a partir del tratamiento. Los niños aumentaron su propia participación en conductas de cuidado a su salud, reduciendo su dependencia del cuidado de otros.

En cuanto a los sentimientos de desesperanza e indefensión generados por un evento estresante como el cáncer (Carver, Pozo-Kaderman, Harris, Noriega, Scheier, Robinson, Ketcham, Moffat, \& Clark, 1994; Grossarth-Maticek \& Eysenck, 1990); niños y padres del presente estudio mejoraron sus habilidades para lidiar con la indefensión y lograron identificar las reacciones emocionales adecuadas ante diversos retos determinados por la enfermedad. Adicionalmente, estas reacciones mostraron más componentes de una comunicación asertiva. 
De igual forma, las conductas intraverbales de los niños y sus padres parecen haber interactuado con el repertorio conductual que empleaban para cuidar su salud. Las personas con "estilos pesimistas" tienden a tener conductas deficientes para su autocuidado y muestran baja adherencia a las prescripciones médicas (Seligman, Reivich, Jaycox, \& Gillham, 1996) En el presente estudio, los participantes lograron identificar sus conductas intraverbales pesimistas y cambiarlas a optimistas, identificando, en ambos casos, las consecuencias emocionales y conductuales de cada patrón de conducta intraverbal. Debe señalarse que los niños lograron generalizar el cambio de conducta intraverbal a su vida cotidiana en aquellos aspectos que tenían impacto en su salud, por ejemplo a situaciones en el ambiente hospitalario, con su familia, en la escuela y con sus amigos.

Así, la intervención denominada "el Juego del Optimismo", mejora el nivel percibido de calidad de vida, incrementa la adherencia terapéutica e incrementa las habilidades sociales en niños con cáncer. Los hallazgos del presente estudio extienden los de otros en el sentido de que las intervenciones de esta naturaleza logran mejorar el repertorio conductual, incrementan las habilidades sociales y mejoran la pertinencia de los argumentos verbales relacionados con la enfermedad y su cuidado en pacientes oncológicos pediátricos (Antoni, Cruess, Cruess, Lutgendorf, Kumar, Ironson, Klimas, Fletcher, \& Schneiderman, 2000; Antoni, Cruess, Cruess, Kumar, Lutgendorf, Ironson, Dettmer, Williams, Klimas, Fletcher, \& Schneiderman, 2000; Bartrop, Lockhurts, Lazarus, Kiloh, \& Penny, 1977; Beck, 1976; Beck, Rush, Shaw \& Emery, 1979; Cruess, Antoni, Cruess, Fletcher, Ironson, Kumar, Lutgendorf, Hayes, Klimas \& Schneiderman, 2000; DeRubeis, Evans, Hollon, Garvey, Grove \& Tuason, 1990; Elkin, Pilkonis, Docherty \& Sotsky, 1988; Ellis, 1962; Galán, 1995; Green, Green \& Santoro, 1988; Glaser \& Kielcolt-Glaser, 1985; Kazdin \& Wilcoxon, 1976; Kuhl, 1981; Nolen-Hoeksema, 1990; Vecina, 2006).

Diversas observaciones informales sobre los cambios generados por el tratamiento revelaron que el participante que no asistía a la escuela empezó a asistir, otro participante inició actividades deportivas destacando en competencias, otro niño inició actividades extraescolares (cursos de inglés y computación a pesar de tener ceguera como consecuencia de los tratamientos), y en los otros tres casos los familiares reportaron mejoría en el ambiente familiar y la convivencia diaria. Por otra parte, cuando los niños requerían hospitalización por complicaciones con su enfermedad, las conductas de adherencia disminuían (como sucede en el caso 6). Diversas variaciones percibidas en sentido clínico sugieren que hace falta considerar en los niños, las diferencias individuales en su desarrollo, la adaptabilidad de conductas según su edad, y la responsividad al tratamiento ya que son variables que afectan las conductas de adherencia.

En virtud de que el paciente oncológico pediátrico interrumpe a menu- 
do su tratamiento psicológico debido a las frecuentes hospitalizaciones se recomienda que la duración de las intervenciones sea, además de breve, integrada por módulos o unidades autocontenidas orientadas a cambios conductuales y emocionales específicos y secuenciados.

Dado que el Sistema Inmune de los sujetos con indefensión presenta disminución de células $T$ y alteraciones en el funcionamiento de las células NK (Bartrop, Lockhurts, Lazarus, Kiloh \& Penney, 1979; Burns \& Seligman, 1989; Irwin, Daniels, Bloom, Smith \& Weiner, 1987; Peterson, Seligman \& Vaillant, 1988) se sugiere que en futuras investigaciones se realicen registros inmunológicos que permitan tener datos sobre el sistema inmune en función de intervenciones psicológicas.

\section{REFERENCIAS}

Aaronson, N.K. (1990) Quality of life assessment in cáncer clinical trials; in J.C. Hollland \& R. Zittoun (Eds). Psychosocial aspects of oncology (pp. 97-111) Germany: European School of Oncology.

Abramson, L.Y, Seligman, M.E.P. \& Teasdale, J. (1978) Learned helplessness in humans: critique and reformulation. Journal of Abnormal Psychology, 87, 49-74.

Altman, E. \& Gotlib, I.H. (1988) The social behaviour of depressed children: an observational study. Journal of Abnormal Child Psychology, 16, 29-44.

Anarte, M. T., López, A. E., Ramírez, C. \& Esteve, R. (2000) Evaluación del Patrón de Conducta Tipo C en Pacientes Crónicos. Anales de Psicología, vol. 16 No.2, 133-141.

Antoni, M., Cruess, S., Cruess, D., Kumar, M., Lutgendorf, S., Ironson, G., Dettmer, E., Williams, J., Klimas, N., Fletcher, M. \& Schneiderman, N. (2000) Cognitive-behavioral stress management reduces distress and 24-hour urinary free cortisol output among symptomatic HIV-infected gay men. Ann Behav Med. 22, 29-37.

Antoni, M., Cruess, D., Cruess, S., Lutgendorf, S., Kumar, M., Ironson, G., Klimas, N., Fletcher, M. \& Schneiderman, N. (2000) Cognitive-behavioral stress management intervention effects on anxiety, 24-hr urinary norepinephrine output, and T-cytotoxic/suppressor cells over time among symptomatic HIV- infected gay men. J. Consult Clin Psychol, 68:31-45.

Antoni, M. \& Scheinderman, N. (1998) HIVIAIDS. In A. Bellack \& M. Hersen (Eds.), Comprehensive clinical psychology (pp237-275). New York: Elsevier Science.

Aspinwall, L., \& Brunhart, S. (2000) What I don't know won't hurt me. En J. Gillham (ed.), The science of optimism and hope: Research essays in honour of MartinE. P. Seligman. (pp 163-200) Philadelphia: Templeton Foundation Press.

Aspinwall, L., Richter, L., \& Hoffman, R.R. (2001) Understanding how optimism works: An examination of optimists' adaptative moderation of belief and behaviour. In E. C. Chang (Ed.), Optimism and pessimism: implications for theory, research, and practice. (pp. 217-238). Washington DC: American Psychological Association.

Bandura, A. (1977) Social learning theory. Englewood Cliffs, N. J. Prentice Hall. 
Baum, A. \& Andersen, B. (2001) Psychosocial interventions for cancer. Washington, DC: American Psychosocial Association.

Barón, G. \& Ordoñez, A. (1998) Oncología Clínica 1: Fundamentos y Patología General. McGraw-Hill, España.

Bartrop, R., Lockhurts, L., Lazarus, L., Kiloh, \& Penny, R. (1977) Decreased Lymphocyte Function After Bereavement. The Lancet, I, 834-836.

Bayés, R. (1990a) Influencia de los factores psicológicos en la longitud de vida de los enfermos oncológicos; en: Martínez Roca editor. Psicología Oncológica. Barcelona: $182-184$

Bayés, R. (1990b). Psicología y cáncer: prevención; en: Martínez Roca, editor. Psicología Oncológica. Barcelona, 47-111.

Beck, A.T. (1976). Cognitive Therapy and the Emotional Disorders. New York: New American Library

Beck, A.T., Rush, A.J., Shaw, B.F., \& Emery, G. (1979) Cognitive Therapy of Depression: A Treatment Manual. New York: Guilford.

Burns, M., \& Seligman, M. (1989) Explanatory style across the lifespan: Evidence for stability over 52 years. Journal of Personality and Social Psychology, 56, 471477.

Cantú, O. A., Cordero, C. R., \& Chávez, R. L. (2003) Análisis Descriptivo de la Calidad de Vida de la Población Oncológica pediátrica Apoyada por una Institución de Asistencia Privada. Tesis de Licenciatura, Facultad de Psicología, UNAM

Carver, C. S., Pozo-Kaderman, C., Harris, S. D., Noriega, V., Scheier, M. F., Robinson, D. S., Ketcham, A. S., Moffat, F. L., y Clark, K. C. (1994). Optimism versus pessimism predicts the quality of women's adjustment to early stage breast cancer. Cancer, 73(4), 1213-1220.

Cruess, S., Antoni, M., Cruess, D., Fletcher, M.A., Ironson, G., Kumar, M., Lutgendorf, S., Hayes, A., Klimas, N. \& Schneiderman, N. (2000) Reduction in herpes simplex virus type 2 antibody titersafter cognitive behavioral stress management and relationships with neuroendocrine function, relaxation skills, and social support in HIV-positive gay men. Psychosomatic Medicine, 62, 828-837.

Danner, D., Snowdon, D., \& Friesen, W., (2001) Positive emotions in early life and longetivity: Findings from the nun study. Journal of Personality and Social Psychology, 80, 804-813.

DeRubeis, R., Evans, M., Hollon, S., Garvey, M., Grove, W. \& Tuason, V. (1990) How do cognitive therapy work? Cognitive change and symptom change in cognitive therapy and pharmacotherapy for depression. Journal of Consulting and Clinical Psychology, 58, 862-869.

Donovan, K., Sanson-Fisher, R.W. \& Redman, S. (1989) Measuring quality of life in cancer patients. Journal of Clinical Oncology, 7 (7): 959-968.

Dweck, C.S., \& Licht, B. (1980) Learned Helplessness and Intellectual Achivement, In J. Garber and M. Seligman, eds., Human Helplessness: Theory and Applications. New York: Academic Press 197-222.

Ellis, A. (1962) Reason and Emotion in Psychotherapy. New York: Lyle Stuart.

Elkin, I., Pilkonis, P., Docherty, J.P., \& Sotsky, S. (1988) Conceptual Methodological Issues in Comparative Studies of Psychotherapy and Pharmacotherapy. American Journal of Psychiatry, 145, 909-917. 
Flores, L. A., \& Bázan, L. E. (2004) Adherencia Terapéutica en Pacientes Oncológico Pediátricos. Tesis de Licenciatura, Facultad de Psicología, UNAM.

Fredickson, B. (1998) What good are positive emotions? Review of General Psychology, 2, 300-319.

Fredickson, B. (2001) The role of positive emotions in Positive Psychology: The broaden-and built theory of positive emotions. American Psychologist, 56, 218-226.

Friedman, A.G. \& Mulhem, R.K. (1992) Psychological aspects of childhood cancer. In B. B. Lahey \& A. E. Kazdin (Eds.) Advances in clinical child psychology (Vol. 14, pp. 165-189). New York: Plenum.

Galán C., S. (1995). Modificación de la función inmune en una población senecta. Tesis de Maestría. Facultad de Psicología. UNAM.

Glaser y Kielcolt-Glaser (1985). Relatively mild stress: depressed celluar immunity in the healthy adults. Behavioral Brain and Science. 8: 401-402.

Green, M.L. Green R.G. \& Santoro, W. (1988). Daily relaxation modifies serum and salivary immunoglobulins and psychophysiologic symptom severity. Biofeedback and Self Regulation. 13 (3): 187-198.

Green, J. \& Shellenberger, R. (1991) The dynamics of health and wellness. A byopsychosocial approach. Fort Worth: Holt, Rinehart \& Winston.

Grossarth-Maticek \& Eysenck (1990) Personality, stress and disease; description and validation of a new inventory. Psychol Reports; 66: 355-373.

Headey, B., \& Wearing, A. (1989) Person ality, life events, and subjective well-being: Toward a dynamic equilibrium model. Journal of Personality and Social Psychology, 57, 731-739.

Hops, H. (1992) Parental depression and child behavior problems: implications for behavioral family intervention; Behavior Change, 9 (3), 126-138.

Hops, H. (1995) Age-and gender-specific effects of parental depression: a comentary; Development Psychology, 31, 428-431.

House, Js; Landis, Kr. \& Umberson D. (1998) Social relationship and health; Science; 241: 540-545

Irwin, M., Daniels, M., Bloom, E.T., Smith, T.L., \& Weiner, H. (1987) Life events, depressive symptoms \& immune function. American Journal of Psychiatry, 144, 437-441.

Isen, A,M., Rosenzweig, A. S., \& Young, M.J., (1991) The influence of positive affect on clinical problem solving. Medical decision Making, 11, 221-227.

Kamen-Siegel, L., Rodin, J., Seligman, M. E., \& Dawyer, C. (1991). Explanatory style and cell-mediated immunity. Health Psychology, 10, 229-235.

Kazdin, A.E., (1982) Single-case research designs: Methods for clinical and applied settings. New York: Oxford University Press. En D. H. Barlow \& M. Hersen (Ed.), Single case experimental designs: Strategies for studying behavior change (pp. 285-371). USA Pergamon General Psychology Series.

Kazdin, A.E. \& Wilcoxon, L.A. (1976) Systematic Desensitization and Nonspecific Treatment Effects: A Methodological Evaluation. Psychological Bulletin, 83, 729-758.

Kiecolt-Glaser R. (1991). Stress and Inmune Function. En R. Ader, D. Felten \& N. Cohen. (Ed) Psychoneuroinmunology (pp 849-868). New York: Academic Press.

Kobasa, S.C. (1979) Stressful live events, personality and health; an inquiry into hardiness. J. Personal \& Soc. Psychol., 37: 1-11. 
Kulh, J. (1981) Motivational and Functional Helplessness: The moderating effect of state versus action-orientation. Journal of Personality and Social Psychology, 40: $155-170$

Maruta, T., Colligan, R., Malinchoc, M., \& Offord K. (2000) Optimists vs. Pessimists: Survival rate among medical patients over a 30 -year period. Mayo Clinic Proceedings, 75, 140-143.

Morris, T. \& Creer, S. (1980) A "type C" for cancer?. Cancer detect prev. 3: 102.

Nolen-Hoeksema, S., (1990) Sex Differences in Depresión: Theory and Evidence. Psychological Bulletin, 101, 259-282.

Ostir, G., Markides, K., Black, S., \& Goodwin, J (2000) Emotional well-being predicts subsequent functional independence and survival. Journal of the American Geriatrics Society, 48, 473-478.

Peterson, C., Seligman, M., \& Vaillant, G. (1988) Pessimistic explanatory style as a risk factor for physical illness: A thirty- five- year longitudinal study. Journal of Personality and Social Psychology, 55, 23-27.

Repetti, R. L., Taylor, S. E., \& Seeman, T. E. (2002). Risky Families: Family social environments and the mental and physical health of offspring. Psychological Bulletin, 128(2), 330-366.

Rutter, M. (1978) Language disorder and infantile autism: En Autism: a reappraisal of conceepts and treatment, ed. M. Rutter \& Schopler, New York: Plenum press, pp. 85-104.

Sánchez- Sosa, J. J. (2002a). Treatment adherence: The role of behavioral mechanisms operating through health care interventions. Revista Mexicana de Psicología, 19(1), 85-92.

Sánchez-Sosa, J. J. (2002b). Health Psychology: Prevention of disease and illness; maintenance of health. UNESCO Encyclopedia of Life Support Systems (EOLSS), Psychology. Oxford, UK, http://www.eolss.net.

Schulman, P., Castellon, C., \& Seligman, M. (1989) Assessing Explanatory Style: The Content Analysis of Verbatim Explanations and the Attributional Style Questionaire. Behavior Research and Therapy, 27 (1989), 505-512.

Seligman, M., \& Elder, G. (1985) Learned Helplessness and Life-Span Development. In A. Sorenson, F. Weinert, and L. Sherrod, eds., Human Development and the Life Course: Miltidisciplinary Perspectives. Hillsdale, N.J.: Erlbaum, 1985, 377-427.

Seligman, M. (1991) Learned optimism. New York: Knopf.

Seligman, M. Reivich, K. Jaycox, L \& Gillham, J. (1996) The optimistic child: a proven program to safeguard children against depression and build lifelong resilience. New York; Harper Perennial.

Seligman, M. (1998) Learned Optimism: How to change your mind and your life. Free Press New York.

Seligman, M. (2002) Authentic Happiness. Free Press New York.

Segerstrom, S., Taylor, S., Kemeny, M., \& Fahey, J. (1998) Optimism is associated with mood, coping, and immune change in response to stress. Journal of Personality and Social Psychology, 74, 1446-1655.

Sheridan, C \& Radmaches, S. (1992) Health Psychology. Singapur: wiley.

Staw, B., Sutton, R., \& Pelled, L. (1994) Employee positive emotion and favorable outcomes at workplace, Organization Science, 5, 51-71. 
Sweeney, P., Anderson, K., \& Bailey, S. (1986) Attributional Style in Depression: A Meta-analytic Review, Journal of Personality and Social Psychology, 50, 974-91.

Temoshok, L. (1987) Personality, copyng style, emotion and cancer: towards and integrative model. Cancer Surv 1987; 6: 545-567.

Vaillant, G. (2000) Adaptative mental mechanisms: Their role in Positive Psychology. American Psychologist, 55, 89-98.

Vaillant, G. (2002) Aging well. New York: Little, Brown.

Valencia, A., (2006) Programa de Apoyo Psicosocial para Niños con Cáncer y sus Familias. Tesis Doctoral. Facultad de Psicología, UNAM

Varni, J. W., Katz, E. R., Seid, M., Quiggins D.J. L., \& Friedman-Bender, A. (1998) The pediatric Cancer Quality of Life Inventory-32 (PCQL-32): I. Reliability and validity. Cancer, 82, 1184-1196.

Vecina, J. M.L. (2006) Emociones positivas. Papeles del Psicólogo, Vol. 27 (1), 9-17.

Wayner, L, Cox, T. \& Mackay, C. (1979) Stress, Inmunity and Cancer. En: Oborne, M; Gruneberg, M. \& Eiser, Jr. editores. Res Psychol Med. Londres 253-259.

Weiner, B., Frieze, I., Kukla, A., Reed, L., Rest, S., \& Rosenbaum, R.M (1971) Perceiving the Causes of Success and Failure. Morristown, N.J.: General Learning Press. 